\title{
Effects of Neuroticism on Partial and Whole Body Reactions under Stress
}

\author{
Kanaka Yatabe ${ }^{1}$, Hiroto Fujiya ${ }^{1}$, Naoko Yui $^{1}$, Keisuke Tateishi ${ }^{1}$, Yuka Murofushi ${ }^{1}$, Fumiko Terawaki ${ }^{1}$, Atsuhiro \\ Yoshida $^{2}$, Hirotaka Yoshioka ${ }^{2}$, Koh Terauchi ${ }^{2}$, Hajime Kobayashi ${ }^{2}$, Takaaki Kudo ${ }^{2}$, Mahiro Ohno ${ }^{2}$, Aya Uchino ${ }^{2}$, \\ Hisao Miyano ${ }^{3}$, Tadasu Oyama ${ }^{4}$ and Haruki Musha ${ }^{1}$ \\ 1. Department of Sports Medicine, St. Marianna University School of Medicine, Kawasaki 216-8511, Japan \\ 2. Department of Sports Medicine, St. Marianna University Graduate School of Medicine, Kawasaki 216-8511, Japan \\ 3. Research Division, National Center for University Entrance Examinations, Tokyo 153-8501, Japan \\ 4. Department of Psychology, College of Humanities and Sciences, Nihon University, Tokyo 156-8550, Japan
}

\begin{abstract}
Effects of neuroticism on partial and whole body reaction times were investigated under stress, or choking conditions in student participants $(N=127)$. We measured simple and choice reaction times of parts of the body and choice reaction times of the whole body in the participants with high and low neuroticism such as depression, cyclic tendency, inferiority feelings, and nervousness under the controlled stress scenario of being in front of people. Results indicated significant differences in reaction times of partial body, discriminative decisions and choice reaction times of participants in the high neuroticism group. Results of whole body reaction times were opposite to that of partial body reactions. However, a two-way factorial analysis of variance indicated neither a significant main effect, nor interactions between reaction times and personality traits. Whole body reactions suggest that the effects of stimulus transduction process from motor commands to motor outputs via motor neurons has a greater effect in the central nervous system than recognition and decision-making. However, it is possible that changes in the intracortical mechanisms related to cognitions and emotions could be correlated in partial body movements with nearly no muscular activity. Our findings suggested that delays indiscrimination, decision-making, and choice times could affect the performance of individuals.
\end{abstract}

Key words: Personality traits, stress, reaction.

\section{Introduction}

Reactions times are important components of motor movements. The movements of body parts in which there is almost no muscular activity have been evaluated during a target-tracking task, by using MEP (motor evoked potential) involuntary finger movements [1]. The authors concluded that increases in excitability of the corticospinal tract were determined by changes in intracortical mechanisms related to cognitions and emotions. Also, in the case of whole body reactions, the stimulus transduction process from motor commands to motor outputs via motor neurons might have a more significant effect in

Corresponding author: Kanaka Yatabe, M.A., assistant professor, research fields: sports psychology, mood and emotion. E-mail: kanaka@marianna-u.ac.jp. the central nervous system than recognition and decision-making. Reaction times are physiologically significant, and are simple, noninvasive tests of peripheral, as well as central neural structures [2].

Many processes involved inhuman mental activities, such as information collection, learning, and thinking, among others, are unconscious processes. This characteristic is distinctively observed in athletics and sports. For these reasons, it is possible that unconscious self-awareness, rather than conscious self-awareness, has a larger impact on individual performance in sports [3, 4].

A previous study has suggested that unrecognizable stimuli could increase the speed of simple movements such as key-pressing reactions. Based on this finding, we examined whether participants high in neuroticism, 
compared to those low in neuroticism, would show differences in discrimination times between partial and complete body movements in terms of psychological and behavioral dimensions.

\section{Purpose}

Stress in sports consist of three dimensions [5]: psychological, physiological and behavioral [6-10]. One of psychological stress is pressure, which is also known as choking. In terms of behavioral dimensions, a decline in the speed of movement resulting from choking has been reported [11]. This study was designed to examine whether differences in psychological states decreased the speed of movements, or increased reaction times, by measuring partial body and whole body reaction times under a stress scenario of being in front of people.

\section{Participants and Method}

Undergraduate students $(N=127)$ participated in an experiment under the controlled stress of being watched by people. We measured simple and choice reaction times in a part of the participant's body, the finger, and the separate reaction times of the left and right sides of the participants' whole body, by using a color detection task. Then, we measured partial and whole body reaction times of the team, by using a crossover design. After the measurement, we divided participants according to their personality trait into two groups, high and low neuroticism, based on their neuroticism score, and conducted a comparative analysis of SRT (simple reaction times), CRT (choice reaction times) and DT (discrimination times) of the two groups of participants.

We used the Japanese version of the Guilford Personality Inventory [12, 13] and conducted between-task comparisons of psychological stress levels resulting from neuroticism. We first extracted four scales of neuroticism: D (depression), C (cyclic tendency), I (inferiority feelings), and $\mathrm{N}$ (nervousness). Each scale had a maximum score of 20 points resulting in a total score 80 points. Based on the mean score of the present population ( $36.4 \pm 2$ SD points) the participants were divided into two groups. The group of the participants with the scores higher than average was regarded as the unstable group, and the other as the stable group [14, 15].

\subsection{Ethical Considerations}

This study was approved by the St. Marianna University School of Medicine Clinical Research Committee (No.1976). The Helsinki Declaration was honored. The surveys were completed anonymously, so that the participants' identities were secured. The survey responses have been maintained under careful conditions. No results containing personal information have been released, and the data will not be used for other purposes.

\subsection{Statistical Analysis}

Of the 127 participants, the results of 120 (72 men and 48 women) were analyzed using a Student's $t$-test for between-groups comparisons and a Paired $t$-test for between-subjects comparison. We also conducted two-way (neuroticism groups $\times$ times) repeated measures ANOVA (analyses of variance) by means of Tukey HSD (honestly significant difference). Follow-up univariate analyses of variance (ANOVAs) were used in cases of significant main effects and interaction. Analyses were carried out using SPSS Statistics (ver. 21.0) and a $P$-value was set $P<0.05$ to determine statistical significance. The criteria for including reaction times in the analysis were reactions exceeding the average reaction time $\pm 3 \mathrm{SD}$, but not exceeding $200 \mathrm{msec}$, which were considered to be respectively indicative of reaction errors and reaction failures and were regarded as missing values.

About the experimental condition in this study, to measure partial body reaction times, we used a reaction time assessment software developed for basic experiments by Okamoto Y. (Response/Reaction Time, Keiso Shobo, Tokyo, Japan) [16, 17] and 
conducted a randomized signal-timing program with approximately one minute duration of simple reaction time measurement (separately for left and right for fingers) and approximately two minutes duration of choice reaction time testing (simultaneous for left and right fingers). We calculated the median reaction times for each condition. To assess SRT, participants pressed the "J" key of a computer keyboard as quickly as possible when the letter "J" appeared in white text on a black computer screen. The time from the onset of the presentation to participant's key press was regarded as their SRT. To assess CRT, participants selected either the "F (left)" key, or the "J (right)" key near the center of the keyboard, and quickly pressed the selected key. The time from the onset of the presentation to participants' decision-making was considered as their CRT.

To measure participants' whole body reaction times, we used a Multi Jump Tester (DKH, Tokyo, Japan). We presented different colored lights to examine differences in participants' sensory DT (discrimination time). Measurement time was approximately one minute per leg. In this method, participants moved their right or left leg from the front of the mat to its top, as quickly as possible in response to a color light that had been selected by the participants from among three colors, which was presented in a randomized order. The trials were randomized three times each for the left and right legs. Therefore, six measurements were conducted. First, participants stood on the mat to which sensors were attached, and one of the three colored lights was randomly turned on in a random order. They simultaneously lifted their leg from the front of the mat and stepped on the mat when the instructed light came on. We regarded the time from the light turning on to participant jumping as the WBCRT (whole body choice reaction time) of that participant; the time from lifting the leg from the mat to landing on the mat was considered their MT (movement time); and the time required for making the decision to jump (decision making time). The MTs could not measure independently, so the WBDTs (whole body discriminative times) were shown in Fig. 1.

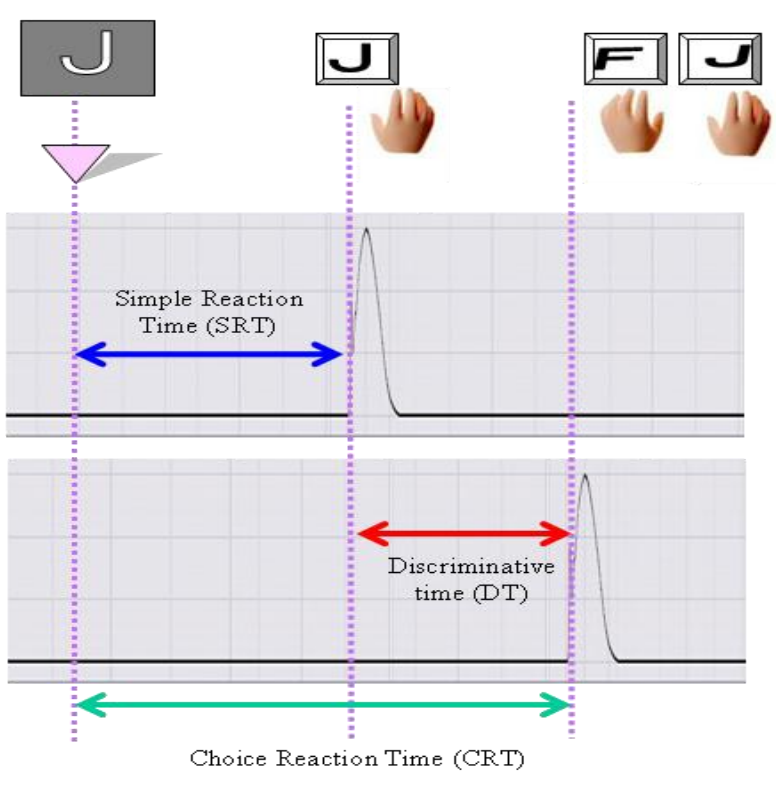

(a) Partial body reaction

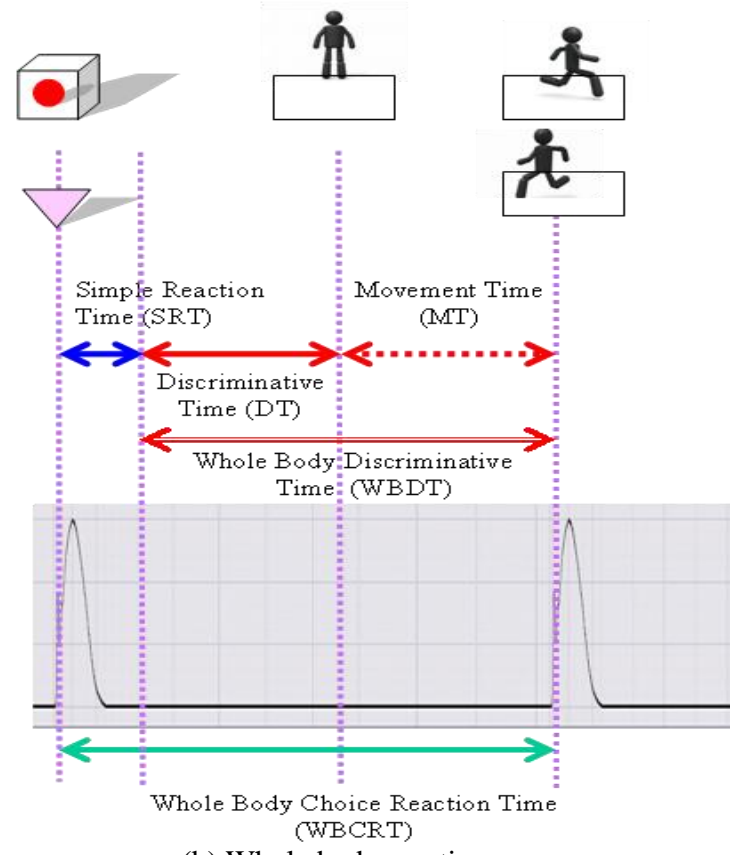

(b) Whole body reaction

Fig. 1 Experimental condition.

DT: The time required for distinction and decision, as reaction start time.

MT: The time required for movement, as muscular contraction time.

WBDTs: The MTs could not measure independently, so the whole body discriminative times (DT + MT $=$ WBDTs). 


\section{Results}

Response times of all participants in the present study indicated that the mean partial body SRT was $268.0 \pm 33.1 \mathrm{msec}$. Similar to most Japanese people, approximately about $90 \%$ of the participants were right handed [18, 19], probably as the results of early training of children's chopsticks-handling in their homes. Although the CRT and DT of the left finger were slightly delayed (CRT: left; $398.1 \pm 51.7$, right; $390.9 \pm 54.3$ msec, DT: left; $130.1 \pm 46.6$, right; 122.9 $\pm 47.7 \mathrm{msec}$ ), there were no statistically significant differences either between the left and the right hands or between men and women (Table 1).

Results also indicated that whole body CRTs were evenly distributed for both the left and right sides of the body and there were no significant differences either in the side of the bodies or in gender.

\subsection{Partial Body Scores of High and Low Neuroticism Groups}

Next partial body tasks were compared between the high neuroticism group and the low neuroticism group. Left CRTs were $389.2 \pm 52.9 \mathrm{msec}$ for the low neuroticism group and $407.2 \pm 49.7 \mathrm{msec}$ for the high neuroticism group. Right CRTs were $383.1 \pm 50.0$ msec for the low neuroticism group and $398.8 \pm 58.1$ msec for the high neuroticism group. Although the high neuroticism group had slightly longer CRTs than the low neuroticism group, there were no significant differences in CRTs between the two groups for left or right fingers as follows, left: $t(116)=-1.908, P=0.059$, right: $t(116)=-1.575, P=0.118$.

However, as can be seen in Fig. 2, there were significant between-group differences $(P<0.05)$ in partial body DTs. Left finger DTs were $120.4 \pm 44.5$ msec for the low neuroticism group and $140.1 \pm 47.5$ msec for the high neuroticism group, $t(116)=-2.334$, $P=0.021$. Right hand DTs were $114.3 \pm 42.2 \mathrm{msec}$ for the low neuroticism group and $131.7 \pm 52.0 \mathrm{msec}$ for the high neuroticism group, $t(116)=-2.000, P=0.048$. We found that high neuroticism group had slightly longer DTs than the low neuroticism group, possible indicative of reduced central processing in the brain.

\subsection{Whole Body Scores of High and Low Neuroticism Groups}

Whole body CRTs of the high and low neuroticism groups showed different trends to that of partial body results (as shown in Fig. 3). There were differences between the left and the right (left: the low neuroticism group $420.7 \pm 119.0 \mathrm{msec}$, the high neuroticism group $366.4 \pm 105.1 \mathrm{msec}$, right: the low neuroticism group $408.1 \pm 109.7 \mathrm{msec}$, the high neuroticism group $370.7 \pm 97.5 \mathrm{msec}$ ). We found that the WBCRTs of the low neuroticism group were longer than those of the high neuroticism group; left:

Table 1 Partial and Whole body reaction times (average \pm standard deviation).

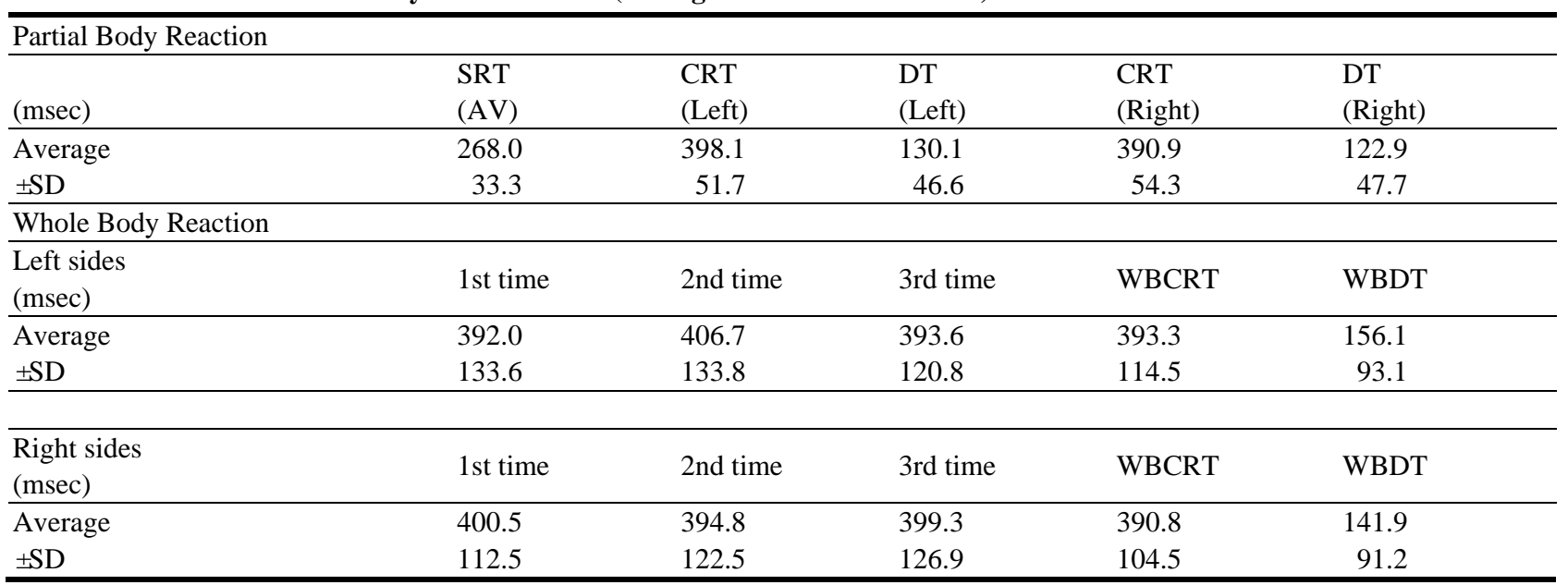




\section{DT}

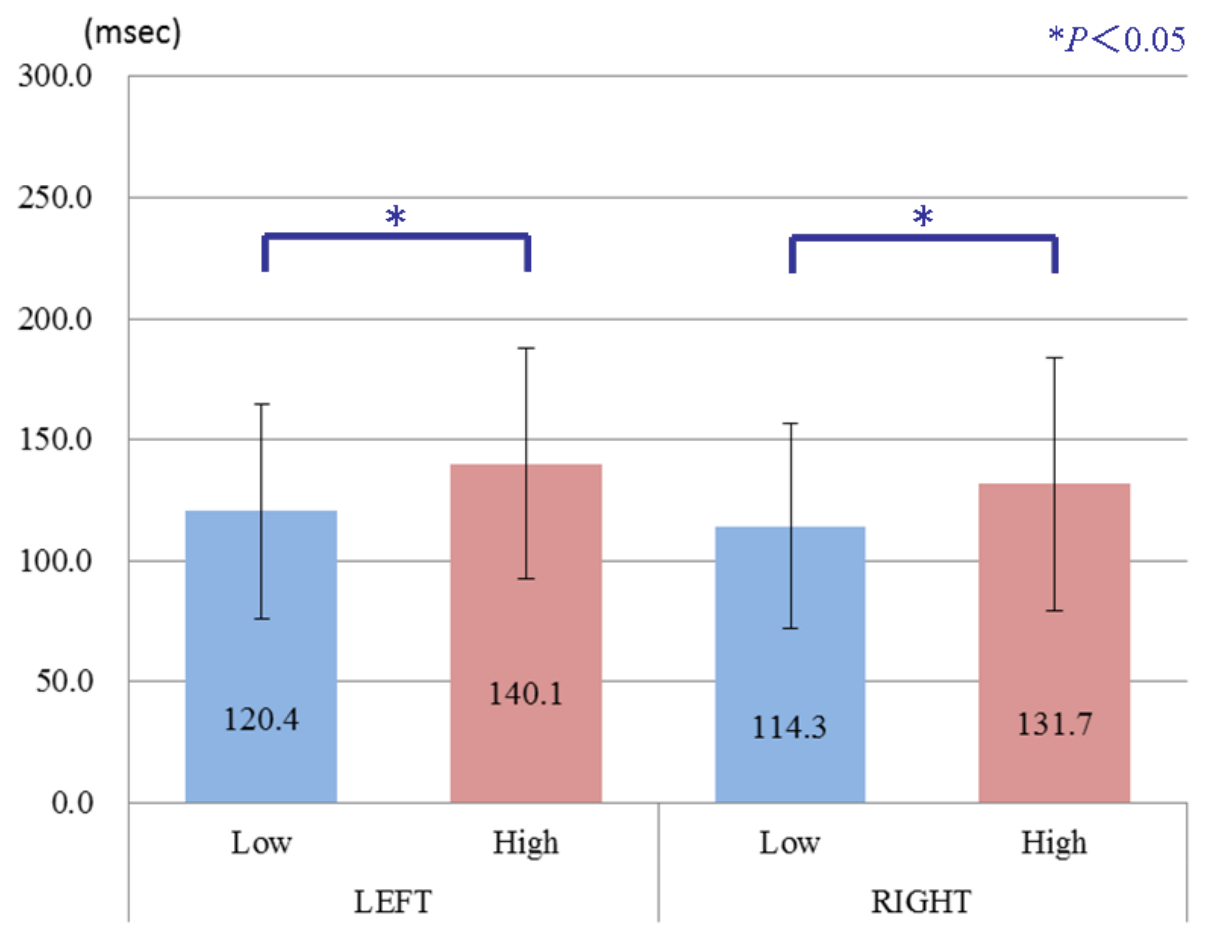

Fig. 2 Partial body discriminative time (by neuroticism traits).

\section{WBCRT}

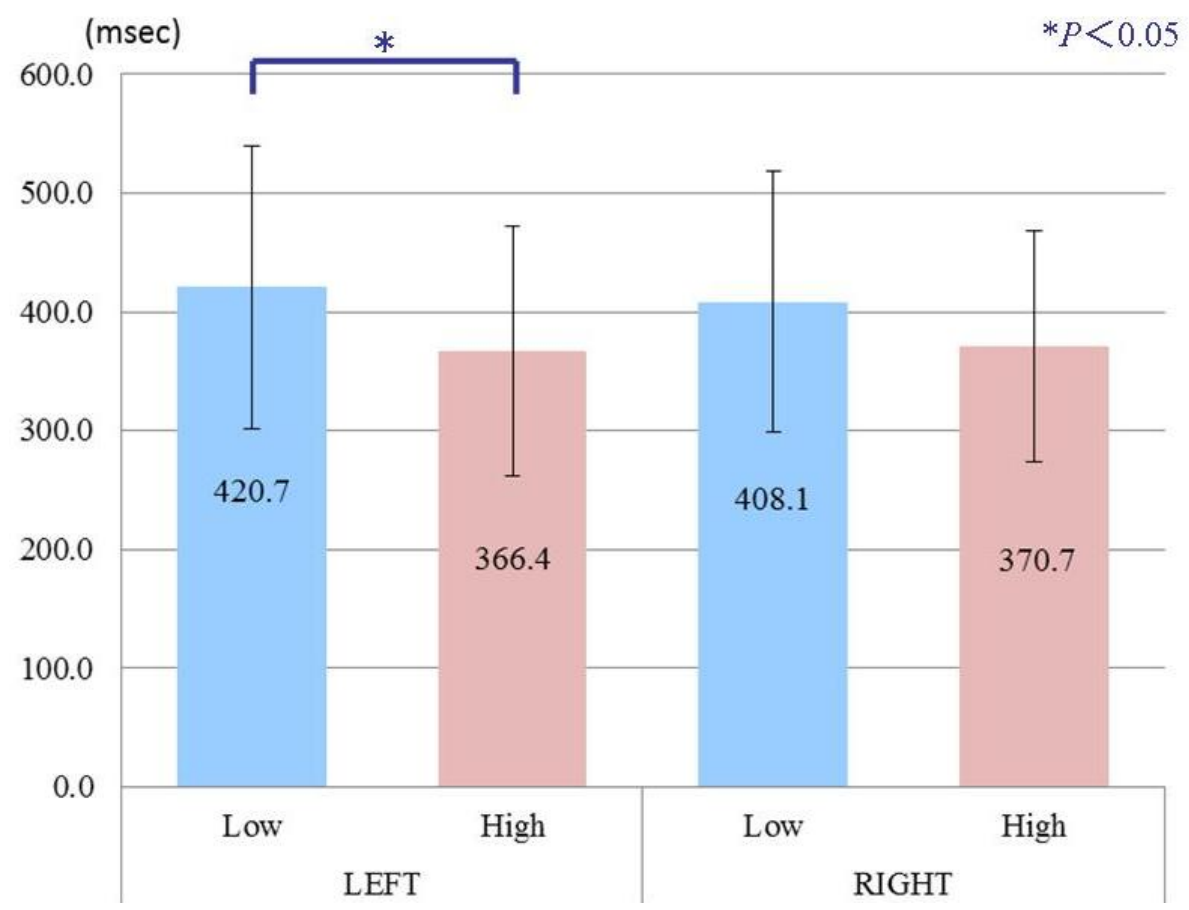

Fig. 3 Whole body choice reaction time (by neuroticism traits). 
$t(113)=2.594, P=0.011$, right: $t(113)=1.925, P=$ 0.057 . This finding suggests opposite effects of neuroticism traits on partial body and whole body reactions.

We conducted two-way factorial ANOVA of whole body reaction measurements, with measurement period and personality traits to examine differences in repeated measurement periods. Results indicated neither a significant main effects nor interactions; left: $F(2,168)=0.082$, right: $F(2,168)=0.595, P \mathrm{~s}>0.05$ (Fig. 4).

It is suggested that slight differences between the left and the right sides of the body were probably related to whether or not it was the dominant leg of the participants. The high neuroticism group had a shorter response time and their performance improved as they repeated the task. The low neuroticism group was more likely to decrease the speed of their performances as the task was repeated. The results were in line with the degrees of performance observed in relation to introversion versus extroversion personality traits [20,21]. The high neuroticism group was more likely to improve their performances as they repeated a task and the low neuroticism group was more likely to perform at their best the first time.

If we review whole body reactions by breaking them down according to the four personality scales of neuroticism (among the four scales), "D (Depression)"

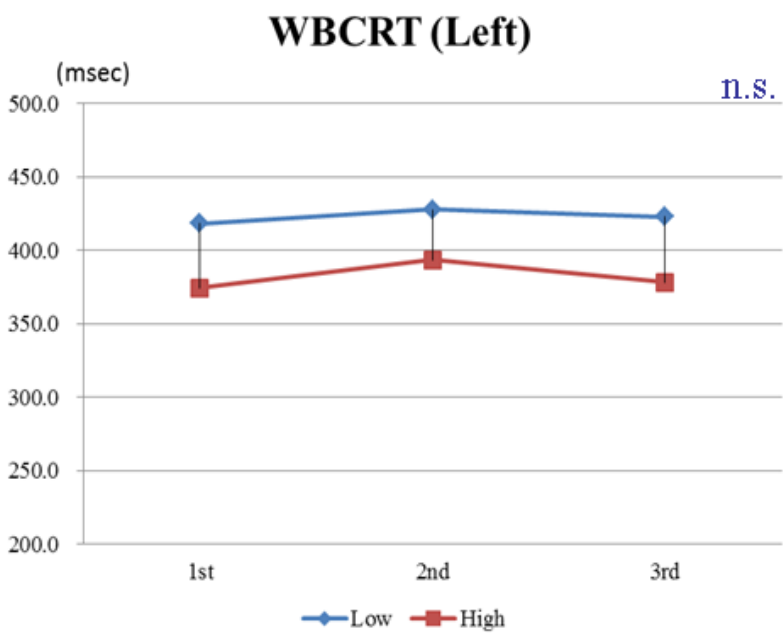

and "N (Nervousness)" scales of the two neuroticism group indicated significant differences by repeated measures ANOVA. (N) scale of the high neuroticism group, indicated a two factor interaction (emotional groups $\times$ period) on the right leg of the body, which was the dominant side of most participants, $F(4,280)$ $=3.442, P=0.035$. Especially in (D) scales on both sides and $(\mathrm{N})$ scale on the right leg of the body, these results indicated that the high neuroticism group tended to be shorter at first time, but were longer at the 2 nd and the 3rd time on the estimated averages.

Differences between the left and the right sides were also observed in whole body DTs (left: low neuroticism group $175.5 \pm 99.6 \mathrm{msec}$, high neuroticism group $135.9 \pm 82.6 \mathrm{msec}$, right: low neuroticism group $153.2 \pm 97.3 \mathrm{msec}$, high neuroticism group $127.9 \pm 83.3 \mathrm{msec}$ ) as see Fig. 5. A Difference $(P<0.05)$ was also observed in the left leg between the two groups, $t(94)=2.107, P=0.038$. We also observed a difference in the same direction for the right leg, which did not reach significance, $t(102)=$ $1.408, P=0.162$. The whole body DTs tended to be opposite of DTs in partial body reactions; the WBDTs of high neuroticism group were shorter than those of low neuroticism group on average.

\section{Discussion}

Results indicated no significant differences between

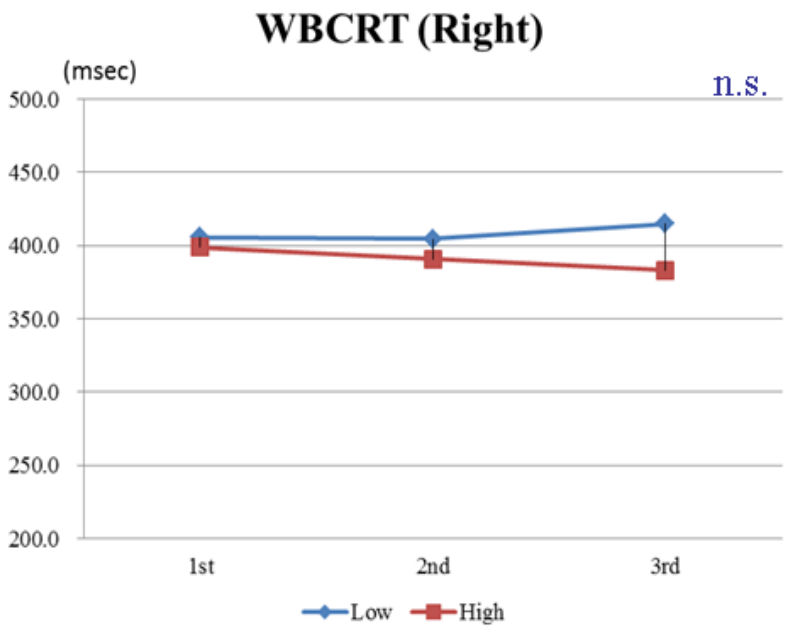

Fig. 4 Whole body choice reaction times (by neuroticism traits). 


\section{WBDT}

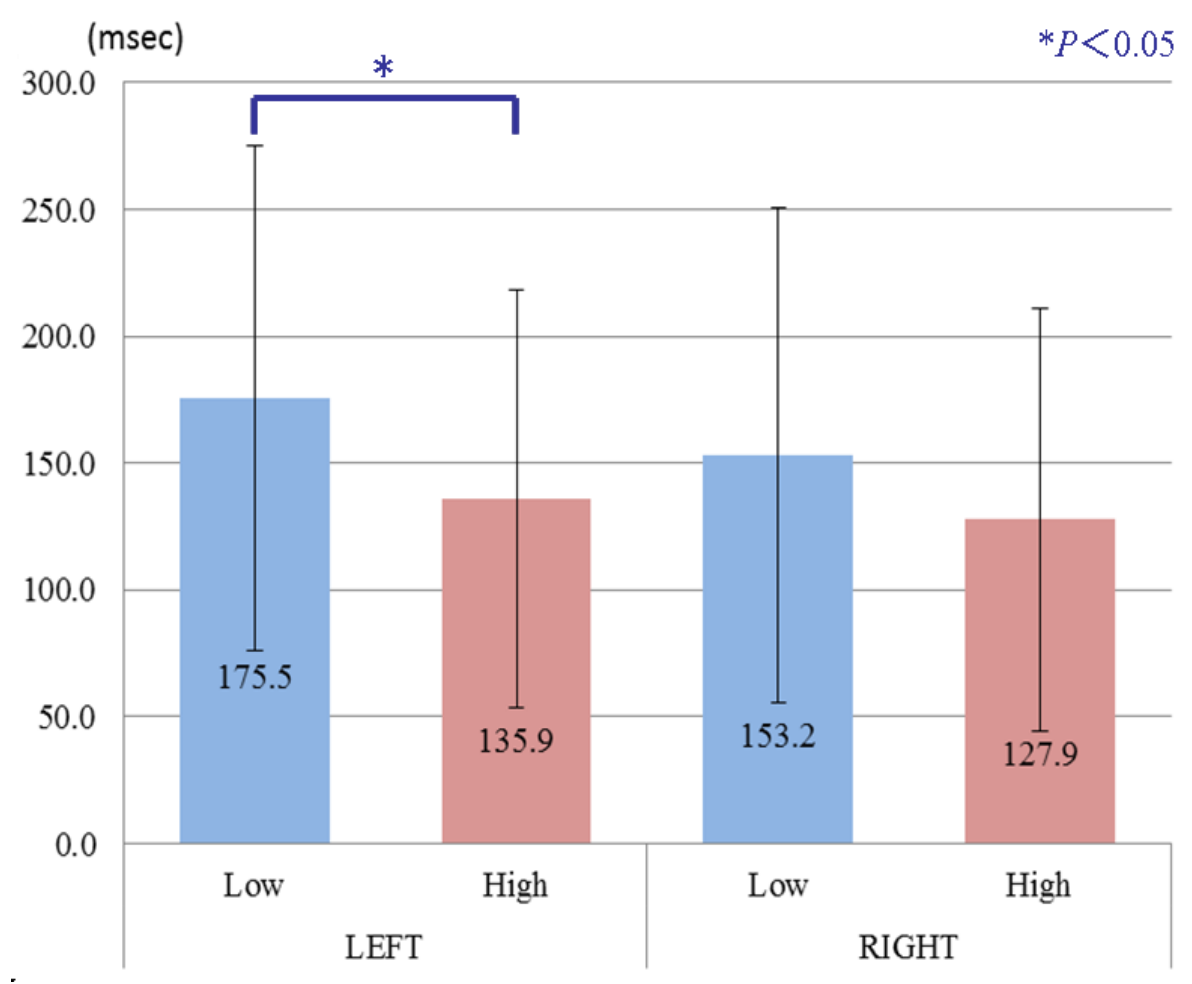

Fig. 5 Whole body discriminative time (by neuroticism traits).

the two neuroticism groups in simple reactions, although the SRTs of the high neuroticism group were slightly shorter than that of the low neuroticism group. There were significant differences between the two neuroticism groups in partial body DTs for both left and right fingers. Although this indicated a weak causative relationship with choking in front of people, we suggest a possible delay in the central processing system of individuals when they are in a nearly unconscious state.

Moreover, the high neuroticism group had a larger standard deviation and a higher degree of dispersion in the semi-interquartile range, which was likely skewed in the direction of longer reaction times (Fig. 6). This might have induced an "uncertainty of movement". Partial body movements, which have nearly no muscular activity, perceptual conflicts such as tiredness, anxiety, and health problems, as well as environmental stressors, such as the need for making social adjustments might influence individuals' performances by decreasing efficiency [22]. Our results corroborated this view. Also, the results supported the findings by Tanaka et al. [1, 23], suggesting that excitability in the corticospinal tract was correlated with changes in the intracortical mechanisms related to cognition and emotions.

In contrast, in the case of whole body reactions, the effects of the stimulus transduction process from motor commands to motor outputs via the pyramidal tract, extrapyramidal tract, and motor neurons was larger than recognition and decision-making in the central nervous system. These results suggest that neuroticism traits do not always have a significant effect on performance [24].

However, if we postulate that there are no differences in the time of stimulus transduction to the whole body, the significant "delays in DTs" observed in the high neuroticism group in the present study 
DT (Left)

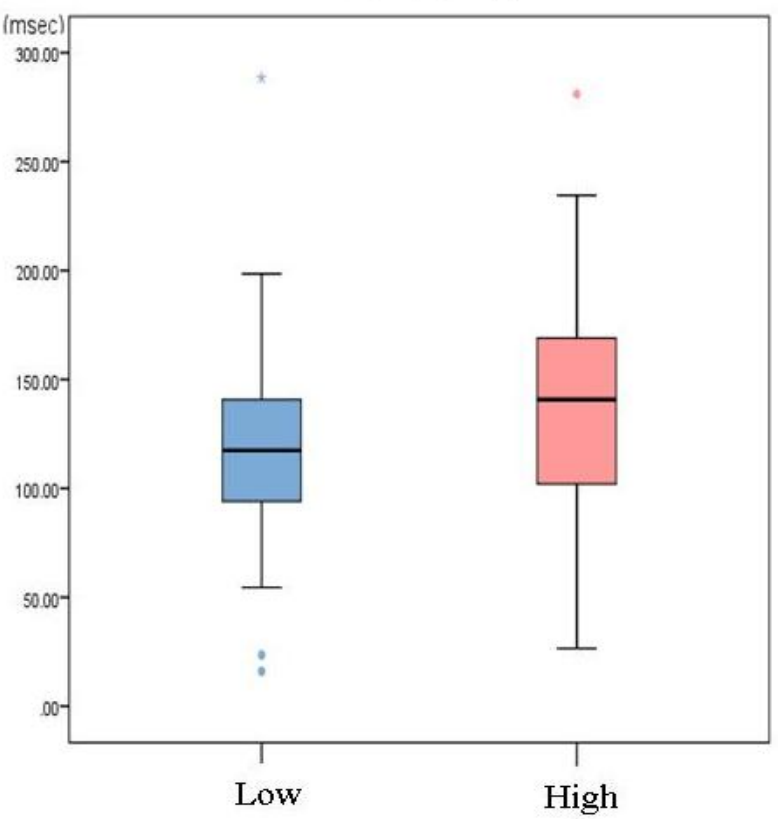

DT (Right)

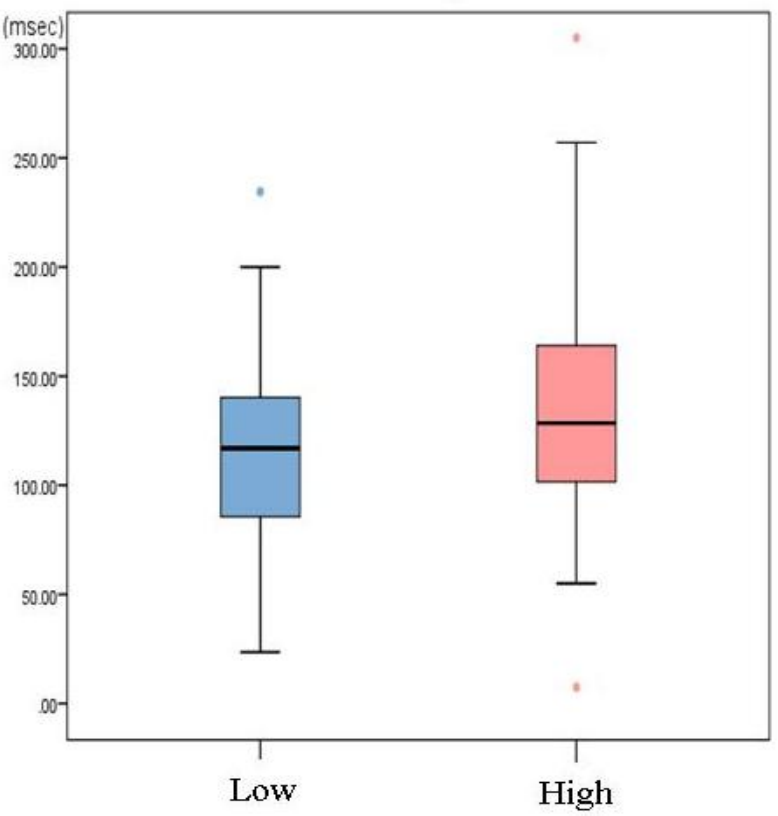

Fig. 6 Dispersion of discriminative time (by neuroticism traits).

might decrease individual performance. In particular, children from 6 to 12 years of age are in a growth period with ongoing development of their nervous system. In the case of such children, the effects of a delayed stimulus transduction time in the motor command output system related to emotions, could have significant effects on their performance.

\section{Future Issues}

It has been suggested with regard to whole body reactions that "emotionally stable groups have a faster whole body reaction time" under psychological stress in the context of personality traits, and that "there is a correlation between pressure and performance" [21, 25-28]. However, our results did not support these findings, because individuals in the present study were facing weak pressure, which might be correlated with attention and concentration for particular types of performance. This could be because an optimal level of tension might have a facilitating effect on choking. This experiment examined differences in participants' whole body reactions under slight pressure, which unlike under strong pressure, might have been insufficient to generate differences in reaction times.

In sports, it has been regarded that reaction time and performance before and after official games differ according to reaction times and neuroticism. However, in the present experiment on whole body reactions, we found the effects of motor control time, or the effects of the stimulus transduction process from the motor command to the motor output via the pyramidal tract, extrapyramidal tract, and motor neurons may have a larger effect. Our findings suggest that in the case of partial body movements, in which there is almost no muscular activity, changes in the intracortical mechanisms related to unconscious cognitions and emotions might be correlated.

\section{Acknowledgements}

This research was supported by a Grant-in-Aid for Scientific Research (No.22700633 and 25350789) from Ministry of Education, Culture, Sports, Science and Technology in Japan and St. Marianna University School of Medicine for helping with data collection on this project. 


\section{Reference}

[1] Tanaka, Y., Funase, K., Sekiya, H., Sasaki., J., and Takemoto, T. 2011. "Multiple EMG Activity and Intracortical Inhibition and Facilitation during a Fine Finger Movement under Pressure." Journal of Motor Behavior 43 (1): 73-81.

[2] Mohan, M., Thombre, D. P., Das, A. K., and Subramanian, N. 1984. "Reaction Time in Clinical Diabetes Mellitus." Indian Journal of Physiology and Pharmacology 28 (4): 311-4.

[3] Masters, R. S. W., Maxwell, J. P., and Eves, F. F. 2009. "Marginally Perceptible Outcome Feedback, Motor Learning and Implicit Processes." Consciousness and Cognition 18 (3): 639-45.

[4] Lewicki, P., Hill, T., and Czyzewska, M. 1992. "Nonconscious Acquisition of Information." American Psychologist 47 (6): 796-801.

[5] Lang, P. J. 1971. "The Application of Psychophysiological Methods to the Study of Psychotherapy and Behavior Modification." In Handbook of Psychotherapy and Behavior Change, edited by Bergin, A., and Grafield, S. New York, NY: Wiley, 75-125.

[6] Ichimura, S. 1965. "Factor Analytical Study on the Traits of Stage Fright in Sports." Japan Journal of Physical Education, Health and Sport Sciences 9 (1): 18-22. (in Japanese)

[7] Arimitsu, K., and Imada, H. 1999. "Situations and Cognitive Appraisals in 'Agari' Experiences: Feature Analyses of 'Agari' Experiences.” The Japanese Journal of Psychology 70 (1): 31-7. (in Japanese)

[8] Wang, J., Marchant, D., and Morris, T. 2004. "Self-consciousness and Trait Anxiety as Predictors of Choking in Sport." Journal of Science and Medicine in Sport 7 (2): 174-85.

[9] Tanaka, Y., and Sekiya, H. 2010. "The Influence of Audience and Monetary Reward on Putting Kinematics of Expert and Novice Golfers." Research Quarterly for Exercise and Sport 81 (4): 416-24.

[10] Mullen, R., Hardy, L., and Tattersall, A. 2005. "The Effects of Anxiety on Motor Performance: A Test of the Conscious Processing Hypothesis." Journal of Sport \& Exercise Psychology 27: 212-25.

[11] Tanaka, Y., and Sekiya, H. 2006. "The Influence of Acute Psychological Stress on Golf Putting." Japanese Journal of Sport Psychology 33 (2): 1-18. (in Japanese)

[12] Guilford, J. P. 1975. "Factors and Factors of Personality." Psychological Bulletin 82 (5): 802-14.

[13] Tsujioka, Y. 1957. "A Factorial Study of the Temperament of Japanese College Male Students by the Yatabe-Guilford Personality Inventory." Psychologia 1 (2): 110-9. (in Japanese)
[14] Luce, R. D. 1986. Response Times: Their Role in Inferring Elementary Mental Organization. Oxford Psychology Series 8 . New York: Oxford University Press, 562.

[15] Welford, A. T. 1977. "Motor Performance." Handbook of the Psychology of Aging 2 (450): 95.

[16] Okamoto, Y. 1982. "A Test of the Fixed-point Property of the Two-state Model for Simple Reaction Time." Japanese Psychological Research 24 (4): 222-4.

[17] Okamoto, Y. 2010. Response/Reaction Time: Psychology VC++ for University Students, Introduction to Program. Tokyo: KeisoShobo. (in Japanese)

[18] Hardyck, C., and Petrinovich, L. F. 1977. "Left-handedness." Psychological Bulletin 84 (3): 385-404.

[19] Maehara, K. 1989. Right-handed Person, Left-handed Science, Handedness, Footedness, Work Eyes, Work Ear. Tokyo: Kodansha. (in Japanese)

[20] Baumeister, R. F. 1984. "Choking under Pressure: Self-consciousness and Paradoxical Effects of Incentives on Skillful Performance." Journal of Personality and Social Psychology 46 (3): 610-20.

[21] Murayama, T., and Sekiya, H. 2012. "Factors Related to Choking under Pressure in Sports and the Relationships among Them." Japan Journal Physical Education, Health and Sport Sciences 57 (2): 595-611. (in Japanese)

[22] O'Relly, and Joseph, P. 1977. "Hana Kai II. A 17-day Dry Saturation Dive at 18.6 ATA: VI. Cognitive Performance, Reaction Time, and Personality Changes." Undersea Biomedical Research 4 (3): 297-305.

[23] Tanaka, Y., and Sekiya, H. 2010. "The Influence of Audience and Monetary Reward on the Putting Kinematics of Expert and Novice Golfers." Research Quarterly for Exercise and Sport 81 (4): 416-24.

[24] Yavuz, H. U., and Oktem, F. 2012. "The Relationship between Depression, Anxiety and Visual Reaction Times in Athletes." Biology of Sport 29 (3): 205-9.

[25] Lenzenweger, M. F. 2001. "Reaction Time Slowing During High-load, Sustained-attention Task Performance in Relation to Psychometrically Identified Schizotypy." Journal of Abnormal Psychology 110 (2): 290-6.

[26] Panayitou, G., and Vrana, S. R. 2004. "The Role of Self-focus, Task Difficulty, Task Self-relevance, and Evaluation Anxiety in Reaction Time Performance." Motivation and Emotion 28 (2): 171-96.

[27] Murayama, T., Tanaka, Y., and Sekiya, H. 2009. "Qualitative Research on the Mechanism of Choking under Pressure." Japan Journal Physical Education, Health and Sport Sciences 54 (2): 263-277. (in Japanese) 
[28] Ciucurel, M. M. 2012. "The Relation between Anxiety, Reaction Time and Performance before and after Sport Competitions." Procedia-Social and Behavioral Sciences
33: 885-889. $\quad$ Accessed May 7, 2015. http://www.sciencedirect.com/science/article/pii/S187704 2812002571 